Reprod. Nutr. Dévelop., 1982, 22 (4), 681-688.

\title{
Variations saisonnières de la cyclicité chez la génisse créole. Influence de la croissance, de l'âge et de l'émotivité
}

\author{
D. GAUTHIER, J. THIMONIER
}

avec la collaboration technique de $\mathrm{H}$. VARO

Station de Recherches zootechniques, I.N.R.A., Domaine Duclos, 97170 Petit-Bourg, France.

Summary. Seasonal variations in creole heifer cyclicity : effect of growth, age and emotivity.

The ovarian and estrous activities in creole heifers have been studied in Guadeloupe in a tropical environment. We carried out two experiments. In the first one, all the heifers from the research station's herd were blood-sampled twice every 2 months at a 10-day interval to determine their ovarian activity. The observations were ranked in order of ascending live weight, and the percentage of heifers showing ovarian activity was calculated for consecutive $10-\mathrm{kg}$ increments in live weight from 115 to $275 \mathrm{~kg}$. The percentage showing ovarian activity $(Y)$ was subjected to probit transformation and related to live weight $(X)$; probit $Y=12.4 \log _{10} X-23.7$. The equation for observations ranked in order of ascending age and the percentage of heifers showing ovarian activity calculated for age increments over 2 consecutive months was : probit $Y=7.06 \log _{10} X-3.9$.

Furthermore, the percentage of cyclic heifers in the herd varied from 40 p. 100 in September 1979 to 0 p. 100 in July 1980 and then to 30 p. 100 in Septembre 1980. The percentage of cyclic heifers at a given period of observation was highly correlated with the mean daily increase in body weight $(r=0.86)$.

In the second experiment, 12 heifers with a high feed level were blood-sampled weekly and checked for estrous daily with an androgenized female with a chin ball mating harness. The mean duration of a " normal cycle " (interval between two estruses : more than 15 but less than 31 days) was 21 days. There was no variation of ovarian activity with the month of the year, but the incidence of silent ovulation was high (23.7 p. 100). The seasonal variation of this percentage is shown.

Female emotivity was also an important factor : emotional heifers had a higher percentage of silent ovulations than unemotional heifers (27 vs 17 p. 100) and a smaller mean daily gain ( 180 vs $280 \mathrm{~g}$ ).

\section{Introduction.}

L'analyse de la répartition des mises-bas des vaches créoles en Guadeloupe fait apparaître clairement une variation saisonnière de la fertilité (Gauthier, résultats non publiés).

Une évolution, au cours de l'année, du pourcentage de femelles présentant œstrus et ovulation peut être invoquée. En zone tempérée et pour des races Euro- 
péennes, Mariana, Mauléon et Solari (1970) ont écarté cette hypothèse. Cependant, en zone tropicale avec des animaux Brahmans, en conditions de " ranching ", Plasse, Warnick et Koger (1970) mettent en évidence une variation saisonnière des ovulations accompagnées d'œstrus et montrent que les femelles émotives ont une moindre aptitude à la reproduction.

Les femelles créoles se trouvant dans des conditions d'élevage proches de celles décrites par ces derniers auteurs, il nous a paru important d'étudier l'activité ovarienne des génisses et d'analyser l'influence de la saison, de la croissance, de l'âge et de "l'émotivité " sur ce paramètre.

\section{Matériel et méthodes.}

Deux expériences ont été réalisées au Domaine expérimental de la Station de Recherches zootechniques de l'INRA en Guadeloupe $\left(16^{\circ} 10^{\prime}\right.$ de latitude Nord et $61^{\circ} 40^{\prime}$ cie longitude Ouest). La moyenne annuelle des précipitations est de $1250 \mathrm{~mm}$. Bien que d'importantes variations entre les années soient observées, une saison des pluies (août à novembre, $800 \mathrm{~mm}$ ) et une période sèche (décembre à juillet, $450 \mathrm{~mm}$ ) peuvent être définies. La température maximale varie entre $27^{\circ} \mathrm{C}$ (janvier) et $32{ }^{\circ} \mathrm{C}$ (août) et la minimale entre $21^{\circ} \mathrm{C}$ et $25^{\circ} \mathrm{C}$. L' L'hygrométrie relative est toujours supérieure à 70 p. 100 . La durée du jour varie entre 11 et $13 \mathrm{~h}$.

$7^{r e}$ expérience. - Elle concerne, du sevrage $(120 \mathrm{~kg})$ à la mise à la reproduction $(250 \mathrm{~kg})$ l'ensemble des génisses de race créole du troupeau de la Station. Du mois de septembre 1979 au mois d'août 1980, les animaux sont maintenus sur pâturage de Pangola (Digitaria decumbens) avec une charge par hectare moyenne de $1600 \mathrm{~kg}$. A partir de septembre 1980, elles sont en stabulation libre sans abri, alimentées à l'auge avec un mélange de Pangola et de Petit foin (Dicanthium caricosum) jusqu'en janvier 1981 puis avec de l'ensilage de Pangola jusqu'en mai 1981. De plus, en stabulation, ces femelles reçoivent une complémentation mélasse-urée, destinée à obtenir un gain moyen quotidien supérieur ou égal à $200 \mathrm{~g}$.

Tous les deux mois, l'activité ovarienne de l'ensemble des génisses est appréciée par l'analyse des niveaux de progestérone plasmatique (Terqui et Thimonier, 1974) de deux prélèvements sanguins collectés à 10 jours d'intervalle (Thimonier, 1978). Deux cent dix observations portant sur 47 femelles ont été ainsi réalisées (de 1 à 8 observations par femelle). Les animaux sont pesés tous les deux mois lors d'un des deux prélèvements.

$2^{e}$ expérience. - Douze génisses non cycliques, de race créole, âgées de 2 à 3 ans et de poids moyen $236 \mathrm{~kg}$ en janvier 1980, sont maintenues en stabulation libre sans abri pendant 15 mois. Elles reçoivent de l'ensilage de Pangola de janvier à juin, puis un mélange de Pangola et de Petit foin distribué en vert de juillet à décembre.

Durant toute l'expérience, les génisses reçoivent une complémentation de $2,5 \mathrm{~kg}$ par tête d'un mélange composé de $85 \mathrm{p}$. 100 de son de blé, $10 \mathrm{p}$. $100 \mathrm{de}$ mélasse, 3 p. 100 d'urée et 2 p. 100 de minéraux. 
Les animaux sont pesés tous les 14 jours. La détection des chaleurs est effectuée à l'aide d'une femelle androgénisée munie d'un harnais marqueur (" chin-ball mating harness ") laissée en permanence avec les génisses (Signoret, 1975). Les marques sont notées tous les matins.

L'activité ovarienne est étudiée par l'analyse du niveau de progestérone plasmatique (Terqui et Thimonier, 1974) d'un prélèvement sanguin effectué une fois par semaine. Tous les deux mois, l'examen cœlioscopique des ovaires par voie abdominale sans mise à jeûn préalable et avec une anesthésie légère (Holland et al., 1981) complète les informations du test précédent et permet d'en définir l'exactitude. Ainsi sur 72 contrôles, pratiqués par laparoscopie, des résultats obtenus par le dosage de la progestérone, la concordance existe dans 71 cas (99 p. 100 d'exactitude).

Des différences individuelles dans la méfiance et l'agressivité des femelles vis à vis de l'homme étant constatées lors des manipulations, une quantification de cette " émotivité " a été tentée par deux fois, à 4 mois d'intervalle. Pour cela dans un parc $(12 \mathrm{~m} \times 4 \mathrm{~m})$ connu des animaux, le comportement, pendant 5 min., de chacun d'eux mis en présence d'un aliment appétent (son, mélasse, urée) est observé par un expérimentateur immobile placé à $6 \mathrm{~m}$ de cet aliment. Dans ces conditions une génisse qui ne consomme pas la nourriture présentée est considérée comme " émotive ".

\section{Résultats.}

\section{Tre expérience}

Cyclicité, poids, âge. - Pour l'ensemble des observations regroupées en fonction du poids vif (classe $10 \mathrm{~kg}$ ), le taux de femelles cycliques varie de 10 à 20 p. 100 entre 120 et $220 \mathrm{~kg}$, augmente rapidement de 20 à 90 p. 100 entre 220 et $240 \mathrm{~kg}$ pour atteindre 100 p. 100 au dessus de $270 \mathrm{~kg}$ (fig. 1).

$\mathrm{Ce}$ taux $(Y)$ après une transformation probit est corrélé au logarithme du poids moyen de la classe $(X), r=0.95$, et est lié à ce poids par l'équation : probit $Y=12.4 \log _{10} X-23.7$. ( $X$ en $\mathrm{kg}$ ).

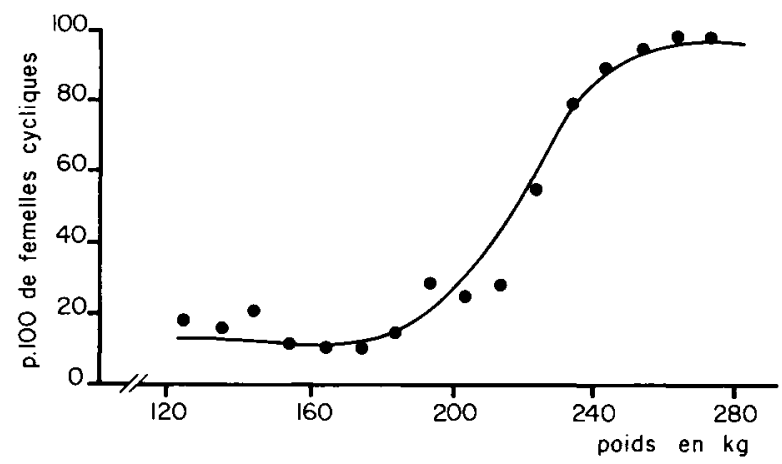

FIG. 1. - Evolution du pourcentage des femelles cycliques par classe de $10 \mathrm{~kg}$ de poids vif. 
Pour l'ensemble des observations regroupées en fonction de l'âge (classe de 2 mois), le taux de femelles cycliques varie de 0 à 40 p. 100 entre 5 et 23 mois, augmente de 40 à 90 p. 100 entre 23 et 27 mois, mais n'atteint 100 p. 100 au-delà de 27 mois que pour des femelles pesant plus de $260 \mathrm{~kg}$. Ce taux (Y) après une transformation probit, est corrélé au logarithme de l'âge moyen de la classe (X), $r=0,80$, et est lié à cet âge par l'équation : probit $Y=7,06 \log _{10} X-3,9(X$ en mois).

Dès l'âge de douze mois, 17 p. 100 des génisses créoles peuvent être cycliques, de 13 à 24 p. 100 selon leur poids. En revanche, à 24 mois et plus, il existe encore des génisses en inactivité ovarienne dans la population étudiée (tabl. 1).

TABLEAU 1

Pourcentage de génisses cycliques par classe d'âge et de poids

\begin{tabular}{cccccc}
\hline & \multicolumn{5}{c}{ Age (mois) } \\
\cline { 2 - 6 } Poids $(\mathrm{kg})$ & $\leq 12$ & $13-18$ & $19-24$ & $>24$ & Total \\
\hline$\leq 150$ & $13(46)$ & $20(5)$ & & & $14(51)$ \\
$151-190$ & $24(29)$ & $8(40)$ & $17(6)$ & & $15(75)$ \\
$191-230$ & & $27(33)$ & $32(28)$ & $0(2)$ & $29(63)$ \\
$>230$ & $17(75)$ & $100(3)$ & $70(9)$ & $100(9)$ & $86(21)$ \\
Total & $20(81)$ & $37(43)$ & $82(11)$ & \\
\hline
\end{tabular}

( ): nombre d'observations.

Cyclicité, variations saisonnières, croissance. - L'âge moyen de l'échantilIon reste constant au cours des deux années d'expérience (environ 14 mois) et les variances sont homogènes. Au cours de la première année, le pourcentage de génisses cycliques varie de 40 en septembre à 0 en juillet (fig. 2). La deuxième année, le pourcentage de femelles cycliques ne varie pas significativement avec la siason (de 30 p. 100 en septembre à 50 p. 100 en mai).

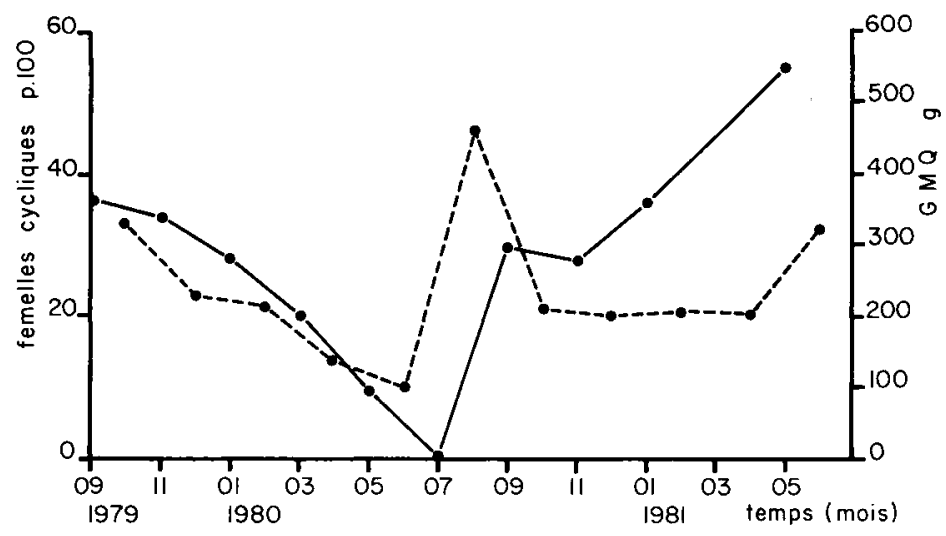

FIG. 2. - Variations saisonnières du pourcentage de génisses cycliques et de leur gain moven quotidien 
Les femelles âgées de moins de 18 mois ne présentent aucune activité ovarienne de mai à septembre, alors que celles âgées de plus de 18 mois ne sont en inactivité que lors du contrôle de juillet.

La variation saisonnière du taux de génisses cycliques suit celle du gain moyen quotidien (fig. 2). Ainsi, ce pourcentage lors d'un contrôle (Y) est une fonction linéaire du logarithme népérien du gain moyen quotidien $(X): Y=23,3$ Log $X-105 ; r=0,86$ ( $X$ en $g$ ). $Y$ est donc nul pour un gain moyen quotidien d'environ $90 \mathrm{~g}$.

Sur 15 femelles ayant atteint au moins l'âge de 14 mois entre septembre et décembre 1979, c'est-à-dire nées à la saison humide précédente, 14 ont ovulé pendant cette période et toutes ont cessé leur activité ovarienne avant mai 1980.

\section{$2^{e}$ expérience}

Oestrus, ovulation, cyclicité. - Lorsque l'étude débute, toutes les femelles sont en ancestrus. La première ovulation est observée à un poids moyen de $269 \pm 5 \mathrm{~kg}$ et à un âge de $28,4 \pm 1,4$ mois. Le poids et l'âge lors du premier œstrus enregistré sont de $271 \pm 6 \mathrm{~kg}$ et de $28,9 \pm 1,5$ mois.

Dans 8 cas sur 12, la première ovulation n'est pas accompagnée d'œestrus ; par la suite 23,7 p. 100 des ovulations se produisent sans qu'aucun comportement de chaleurs ne soit détecté, avec des variations individuelles importantes de 11 à 40 p. 100 . En revanche, seuls 2 p. 100 des œstrus ne sont pas accompagnés d'ovulation. Le pourcentage de génisses présentant au moins une ovulation dans le mois augmente de 0 à 100 de janvier à mai puis n'évolue plus de façon significative par la suite. Le pourcentage d'ovulations non accompagnées d'œstrus est plus élevé pendant les mois de mars, avril, mai, juin $(28,5$ p. 100$)$ que pendant le reste de l'année $(12,0$ p. $100, P<0,01)$. L'intervalle entre deux œestrus consécutifs varie de 6 à 85 jours pour l'ensemble des observations sans qu'un effet saisonnier puisse être mis en évidence. L'étude de la distribution de ces intervalles montre trois populations :

- 11 p. 100 de ces intervalles ont une durée inférieure à 15 jours. Ils correspondent à des œestrus non accompagnés d'ovulation et à des cycles ovariens courts.

- 68 p. 100 ont une durée comprise entre 16 et 30 jours. Ils sont associés dans tous les cas à des œestrus accompagnés d'ovulation ; leur durée moyenne est de $21,0 \pm 1,3$ jours (de 19,2 à 23,4 jours selon les femelles, les différences entre femelles n'étant pas significatives).

- 21 p. 100 ont une durée supérieure à 30 jours. Ils sont dans 80 p. 100 des cas des multiples de la population précédente.

Oestrus, émotivité et croissance. - Les femelles soumises aux deux tests d'émotivité ont été classées les deux fois dans la même catégorie. Le test étant donc répétable, elles sont réparties en deux groupes : 5 sont ainsi qualifiées d'émotives et 7 de non émotives. En début d'expérience les âges et poids moyens respectifs sont de $23.0 \pm 1,5$ mois et $256 \pm 7 \mathrm{~kg}$ (femelles non émotives) et de $28,5 \pm 1,4$ mois et $275 \pm 5 \mathrm{~kg}$ (femelles émotives ; $P>0,1$ ).

Les pourcentages d'ovulations silencieuses sont respectivement de 27 et de 17 p. $100(P<0,05)$ pour les femelles émotives ou non. De plus, les femelles 
émotives ont un gain moyen quotidien pendant l'expérience inférieur à celui des autres (180 vs $280 \mathrm{~g} ; \mathrm{P}<0,01)$. Le pourcentage individuel d'ovulations silencieuses est corrélé avec le gain moyen quotidien $(r=-0,71 ; P<0,01)$.

\section{Discussion.}

Chez les génisses créoles, l'activité ovarienne s'établit à 25 mois environ. Cet âge est plus proche de celui décrit pour Bos indicus que pour Bos taurus (Plasse, Warnick et Koger, 1968 ; Roy, Gillis et Shotton, 1975 ; Laster et al., 1979).

Leur poids est alors de $230 \mathrm{~kg}$ soit entre 60 et 65 p. $100 \mathrm{du}$ poids des adultes ce qui traduit une absence de précocité (Roy, Gillies et Shotton, 1975). Le poids vif d'une femelle étant le facteur primordial du déclenchement de sa puberté (Joubert, 1963 ; Wiltbank, Kasson et Ingalls, 1969 ; Short et Bellows, 1971) I'initiation de la cyclicité obtenue à un âge moyen élevé chez la génisse créole serait la conséquence de son absence de précocité physiologique et de sa faible croissance $(270 \mathrm{~g} / \mathrm{j})$.

Bien plus, le taux de cyclicité d'un groupe de génisses est lié au gain moyen quotidien $\left(r^{2}=0,74\right)$. II en résulte, dans nos conditions d'expérience, une variation saisonnière du pourcentage de femelles en activité ovarienne. L'existence d'une telle variation saisonnière chez les bovins a déjà été décrite par Plasse, Warnick et Koger (1968), Thimonier et Pelot (1976). Le niveau d'alimentation est probablement le facteur principal impliqué dans ces variations.

Ainsi lorsque le gain moyen quotidien est inférieur à $90 \mathrm{~g}$, les génisses créoles sont en ancestrus. En revanche, lorsque les conditions alimentaires restent satisfaisantes ( $2^{\mathrm{e}}$ expérience) il n'existe pas d'arrêt de l'activité ovarienne comme l'ont aussi montré Mariana, Mauléon et Solari (1970). Néanmoins, dans nos observations, le pourcentage mensuel d'ovulations silencieuses est, lui, variable. Température (Plasse, Warnick et Koger, 1970), photopériode (Tucker et Oxender, 1980), démarrage de l'activité ovarienne (Terqui et al., 1981) peuvent être les facteurs responsables de cette variation.

Le pourcentage d'ovulations silencieuses est élevé $(23,7$ p. 100) et plus proche de celui indiqué pour Bos indicus que pour Bos taurus (Kidder, Barret et Casida, 1952 ; Plasse, Warnick et Koger, 1970). Une moindre sensibilité aux œstrogènes du système nerveux central chez la génisse créole, comme cela a été montré chez la Brahman ovariectomisée (Rhodes et Randel, 1978), pourrait expliquer l'absence d'œestrus lors d'une ovulation et/ou l'augmentation de l'erreur par défaut de la méthode de détection des chaleurs utilisée (5 p. 100, Signoret, 1975).

Comme le confirment nos résultats, l'émotivité est un important facteur limitant de la productivité chez certains types de bovins (Plasse, Warnick et Koger, 1970 ; Gupta et Mishra, 1979). Ces auteurs n'ont cependant qu'une appréciation subjective de ce comportement. Le test décrit dans ce travail apporte quelques éléments de quantification.

La distribution de la durée des cycles est identique à celle observée chez Bos taurus (Chapman et Casida, 1937) et chez Bos indicus (Plasse, Warnick et Koger, 1970 ; Madalena et Hinojosa, 1976). Les cycles courts correspondent à la mise en place de la cyclicité. Leur détection par dosage de la progestérone dans des prélè- 
vements sanguins effectués à 10 jours d'intervalle n'est pas toujours possible ; de ce fait l'âge auquel l'activité ovarienne s'établit chez la génisse créole est légèrement surestimé ( $1^{\text {re }}$ expérience). La durée des cycles normaux (Madalena et Hinojosa, 1976) est identique à celle de l'ensemble des bovins (Salisbury, Van Demark et Lodge, 1978). Les cycles longs résultent dans la plupart des cas d'une ou plusieurs ovulations silencieuses.

\section{Conclusion.}

Cette étude nous permet de définir l'âge et le poids à l'établissement de la cyclicité chez les génisses créoles. Ainsi par leurs caractéristiques de reproduction, âge et poids élevés à la puberté, pourcentage important d'ovulations silencieuses, ces animaux s'apparentent plus aux zébus qu'aux taurins. II existe des variations saisonnières de l'activité ovarienne cyclique, le niveau d'alimentation étant le facteur primordial. La mise à la reproduction de ces génisses pendant une période de faible croissance nécessitera donc l'apport d'une complémentation et/ou l'utilisation d'un traitement d'induction de l'ovulation.

Reçu en janvier 1982.

Accepté en mars 1982.

\section{Références}

CHAPMAN A. B., CASIDA L. E., 1937. Analysis of the variation of the sexual cycle and some of its component phases with special reference to cattle. J. agric. Res., 54, 417-422.

GUPTA S. C., MISHRA R.R., 1979. Effect of dairy temperament on milking ability of Karan Swiss cows. Indian J. Dairy Sci., 32, 32-36.

HOLLAND E. J., BINDON B. M., PIPER L. R., THIMONIER J., CORNISH K. A., RADFORD H. M., 1981. Endoscopy in cattle : techniques for ovarian examination by the paralumbar and mid ventral routes. Anim. Reprod. Sci., 4, 127-135.

JOUBERT D. M., 1963. Puberty in female farm animals. Anim. Breed. Abstr., 31, 295-306.

KIDDER H. E., BARRETT G. R., CASIDA L. E., 1952. A study of ovulation in six families of Holstein-Friesians. J. Dairy Sci, 35, 436-440.

LASTER D. B., SMITH G. M., CUNDIFF L. V., GREGORY K. E., 1979. Characterization of biological types of cattle (cycle II). II. Postweaning growth and puberty of heifers. J. anim. Sci., 48, 500-508.

MADALENA F. E., HINOJOSA C., 1976. Reproductive performance of zebu compared with charolais-zebu females in a humid tropical environment. Anim. Prod., 23, 55-62.

MARIANA J. C., MAULÉON P., SOLARI A., 1970. Variations saisonnières de l'œestrus et de l'ovulation chez des génisses de race Française frisonne pie-noire et Charolaise. Analyse des séquences d'ovulations. Ann. Biol. anim. Bioch. Biophys., 10, 567-573.

PLASSE D., WARNICK A. C., KOGER M., 1968. Reproductive behavior of Bos indicus females in a subtropical environment. I. Puberty and ovulation frequency in Brahman and Brahman $\mathrm{x}$ British heifers. J. anim. Sci., 27, 94-100.

PLASSE D., WARNICK A. C., KOGER M., 1970. Reproductive behavior of Bos indicus females in a subtropical environment. IV. Length of estrous cycle, duration of estrus, time of ovulation, fertilization and embryo survival in grade Brahman heifers. J. anim. Sci., 30, 63-72.

RHODES R. C., RANDEL R. D., 1978. Reproductive studies of Brahman cattle. I. Behavioral effect of various dose levels of estradiol-17 $\beta$ upon ovariectomized Brahman, Brahman $x$ Hereford and Hereford cows. Theriogenology, 9, 429-435. 
ROY J. H. B., GILLIES C. M., SHOTTON S. M., 1975. Factors affecting first oestrus in cattle and their effects on early breeding, 128-142. In TAYLER J. C., The early ca/ving of heifers and its impact on beef production. Commission of the European Communities, Brussels.

SALISBURY G. W., VAN DEMARK N. L., LODGE J. R., 1978. Management factors that affect the reproductive efficiency of the bull, 733-779. In Physiology of reproduction and artificial insemination of cattle. W. H. Freeman and Co., San Francisco.

SHORT R. E., BELLOWS R. A., 1971. Relationships among weight gains, age at puberty and reproductive performance in heifers. $J$. anim. Sci., 32, 127-131.

SIGNORET J. P., 1975. Nouvelle méthode de détection de l'oestrus chez les bovins. Ann. Zootech., 24, 125-127.

TERQUI M., CHUPIN D., GAUTHIER D., PEREZ N., PELOT J., MAULÉON P., 1981. Influence of management and nutrition on post partum endocrine function and ovarian activity in cows. Munich. C.E.E. Congress (in press).

TERQUI M., THIMONIER J., 1974. Nouvelle méthode radioimmunologique rapide pour l'estimation du niveau de progestérone plasmatique. Application pour le diagnostic précoce de la gestation chez la brebis et la chèvre. C.R. Acad. Sci. Paris, Sér. D., 279, 1109-1112.

THIMONIER J., 1978. L'activité ovarienne chez les bovins. Moyens d'étude et facteurs de variations. Ann. Méd. vét., 122, 81-92.

THIMONIER J., PÉLOT J., 1976. Maîtrise de la reproduction chez les vaches allaitantes. Bull. G.T.V., 4, 1-6.

TUCKER H. A., OXENDER W. D., 1980. Seasonal aspects of reproduction, growth and hormones in cattle and horses. Progr. reprod. Biol., 5, 155-180.

WILTBANK J. N., KASSON C. W., INGALLS J. E., 1969. Puberty in crossbred and straightbred beef heifers on two levels of feed. J. anim. Sci., 29, 602-605. 